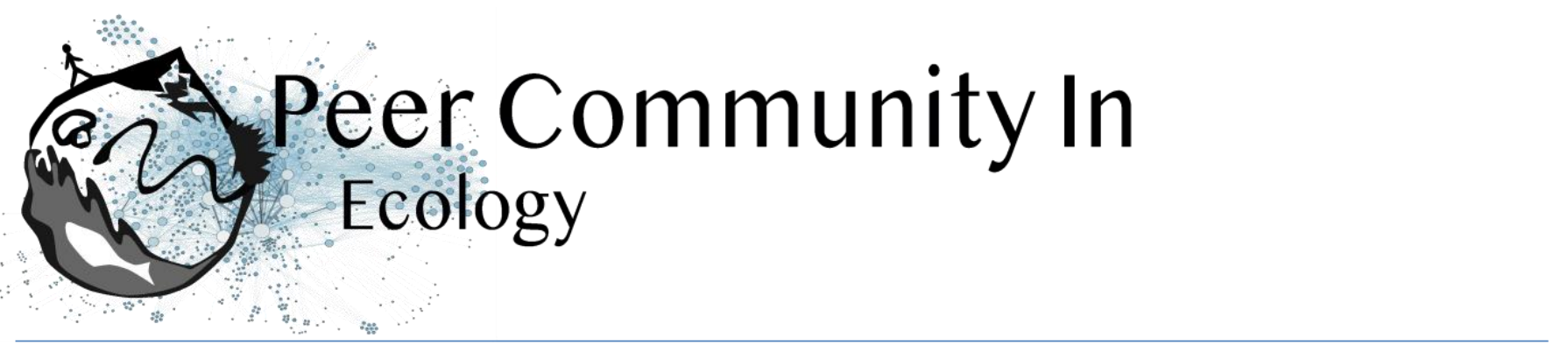

\title{
The search for sex pheromones in malaria mosquitoes
}

\author{
Niels Verhulst based on reviews by Marcelo Lorenzo and 1 anonymous \\ reviewer
}

\section{A recommendation of:}

No Evidence for Long-range Male Sex Pheromones in Two Malaria Mosquitoes

Serge Bèwadéyir Poda, Bruno Buatois, Benoit Lapeyre, Laurent Dormont,

\section{Open Access} Abdoulaye Diabaté, Olivier Gnankiné, Roch K. Dabiré, Olivier Roux(2022), bioRxiv, 2020.07.05.187542, ver. 6 peer-reviewed and recommended by Peer Community in Ecologyhttps://doi.org/10.1101/2020.07.05.187542

Published: 12 January 2022

Copyright: This work is licensed under the Creative Commons Attribution-NoDerivatives 4.0 International License. To view a copy of this license, visit http://creativecommons.org/licen ses/by-nd/4.0/

\section{Recommendation}

Pheromones are used by many insects to find the opposite sex for mating. Especially for nocturnal mosquitoes it seems logical that such pheromones exist as they can only partly rely on visual cues when flying at night. The males of many mosquito species form swarms and conspecific females fly into these swarms to mate. The two sibling species of malaria mosquitoes Anopheles gambiae s.s. and An. coluzzii coexist and both form swarms consisting of only one species. Although hybrids can be produced, these hybrids are rarely found in nature. In the study presented by Poda and colleagues (2022) it was tested if long-range sex pheromones exist in these two mosquito sibling species.

In a previous study by Mozūraites et al. (2020), five compounds (acetoin, sulcatone, octanal, nonanal and decanal) were identified that induced male swarming and increase mating success. Interestingly these compounds are frequently found in nature and have been shown to play a role in sugar feeding or host finding of An. gambiae. In the recommended study performed by Poda et al. (2022) no evidence of long-range sex pheromones in $A$. gambiae s.s. and An. coluzzii was found. The discrepancy between the two studies is difficult to explain but some of the methods varied between studies. Mozūraites et al. (2020) for example, collected odours from mosquitoes in small 1 I glass bottles, where swarming is questionable, while in the study of Poda et al. (2022) $50 \times 40 \times 40 \mathrm{~cm}$ cages were used and swarming observed, although most swarms are normally larger. On the other hand, some of the analytical techniques used in the Mozūraites et al. (2020) study 
were more sensitive while others were more sensitive in the Poda et al. (2022) study. Because it is difficult to prove that something does not exist, the authors nicely indicate that "an absence of evidence is not an evidence of absence" (Poda et al., 2022). Nevertheless, recently colonized species were tested in large cage setups where swarming was observed and various methods were used to try to detect sex pheromones. No attraction to the volatile blend from male swarms was detected in an olfactometer, no antenna-electrophysiological response of females to male swarm volatile compounds was detected and no specific male swarm volatile was identified.

This study will open the discussion again if (sex) pheromones play a role in swarming and mating of malaria mosquitoes. Future studies should focus on sensitive real-time volatile analysis in mating swarms in large cages or field settings. In comparison to moths for example that are very sensitive to very specific pheromones and attract from a large distance, such a long-range specific pheromone does not seem to exist in these mosquito species. Acoustic and visual cues have been shown to be involved in mating (Diabate et al., 2003; Gibson and Russell, 2006) and especially at long distances, visual cues are probably important for the detection of these swarms.

\section{References}

Diabate A, Baldet T, Brengues C, Kengne P, Dabire KR, Simard F, Chandre F, Hougard JM, Hemingway J, Ouedraogo JB, Fontenille D (2003) Natural swarming behaviour of the molecular $\mathrm{M}$ form of Anopheles gambiae. Transactions of The Royal Society of Tropical Medicine and Hygiene, 97, 713-716. https://doi.org/10.1016/S0035-9203(03)80110-4

Gibson G, Russell I (2006) Flying in Tune: Sexual Recognition in Mosquitoes. Current Biology, 16, 1311-1316. https://doi.org/10.1016/j.cub.2006.05.053

Mozūraitis, R., Hajkazemian, M., Zawada, J.W., Szymczak, J., Pålsson, K., Sekar, V., Biryukova, I., Friedländer, M.R., Koekemoer, L.L., Baird, J.K., Borg-Karlson, A.-K., Emami, S.N. (2020) Male swarming aggregation pheromones increase female attraction and mating success among multiple African malaria vector mosquito species. Nature Ecology \& Evolution, 4, 13951401. https://doi.org/10.1038/s41559-020-1264-9

Poda, S.B., Buatois, B., Lapeyre, B., Dormont, L., Diabate, A., Gnankine, O., Dabire, R.K., Roux, O. (2022) No evidence for long-range male sex pheromones in two malaria mosquitoes. bioRxiv, 2020.07.05.187542, ver. 6 peer-reviewed and recommended by Peer Community in Ecology. https://doi.org/10.1101/2020.07.05.187542

\section{Cite this recommendation as:}

Niels Verhulst (2022) The search for sex pheromones in malaria mosquitoes. Peer Community in Ecology, 100091. https://doi.org/10.24072/pci.ecology.100091

\section{Reviews}




\section{(4.7) $\mathrm{PCl}$}

Evaluation round \#2

DOI or URL of the preprint: https://doi.org/10.1101/2020.07.05.187542

Version of the preprint: 3

Author's Reply, None

Download author's replyDownload tracked changes file Decision by Niels Verhulst, 13 Dec 2021

\section{Dear authors}

The two reviewers are positive about the revisions that were made. I agree with the second reviewer that it would be good to describe the possible limitations in a bit more detail. It is surprising that no volatiles were detected from a group of mosquitoes when compared to an empty cage and different methods may change this.

Kind regards

Niels Verhulst

\section{Reviewed by Marcelo Lorenzo, 02 Nov 2021}

I am satisfied with the answers given by the authors and their revised version. I have no additional comments or suggestions.

\section{Reviewed by anonymous reviewer, 12 Dec 2021}

Dear Dr. Verhulst, dear Niels,

I appreciate the effort of the authors in improving the readability of the manuscript. The results of the behavioural assays are a good contribution for the literature and the authors acknowledge the limitations of the study considering that a higher number of swarming males could be needed in the experiments, and that this perhaps explains why the authors do not detect differences in volatiles compounds when comparing an empty box with a box containing the swarming males. I think the fact that the authors used quite a high flow could be an alternative explanation for the fact that they don't detect any differences between an empty box and a box containing the swarming males. It is unlikely that the insects do not emit any volatiles, even when these are not exploited by females as pheromones. Moreover, employing synthetic air or pumping air from a compressed air line with the use of additional (charcoal) filters could lead to cleaner sample collection than pumping air from the environment. I think the manuscript could still benefit from a more open discussion on these aspects, and the overall conclusion should be toned down.

Detailed comments:

Line 220, 236 and elsewhere: it should read dynamic headspace collection Line 221, 390: the term extraction is incorrect 


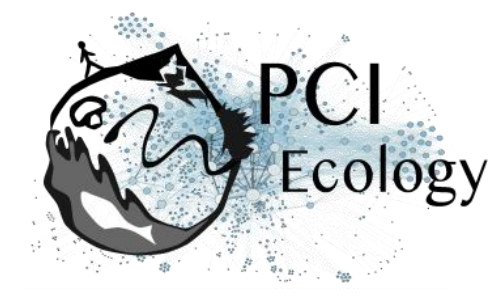

\author{
Evaluation round \#1 \\ DOI or URL of the preprint: https://doi.org/10.1101/2020.07.05.187542
}

\title{
Version of the preprint: 2
}

\author{
Author's Reply, None \\ Download author's replyDownload tracked changes file \\ Decision by Niels Verhulst, 22 Jul 2021
}

Dear authors

Your manuscript has been reviewed and both reviewers are enthusiastic about the topic and the methods used. However, they made good suggestions on how to improve the structure and content of the manuscript. Espescially the selection and analysis of volatiles requires clarification and it would be good to include the underlying data.

Looking forward to your revision.

Kind regards

Niels Verhulst

\section{Reviewed by Marcelo Lorenzo, 30 Jun 2021}

Poda et al-PCl

The manuscript by Poda and collaborators focuses on a long-debated topic: the existence of communication signals potentially mediating mating in Anopheles gambiae and An.

coluzzii mosquitoes. The manuscript is mostly focused on replicating the study by Mozuraitis and collaborators and extending this with additional experimental approaches. Based on their results, male mosquitoes of the two species would lack volatile signals promoting female attraction, contradicting the previous report. Therefore, authors have shown that the results and conclusions by Mozuraitis and collaborators should be considered as potentially flawed and require further replication by third parties.

I suggest the authors have the English language style checked in order to avoid some slight distractions caused by small issues here and there.

Specific comments follow below:

Introduction

Authors should avoid dedicating such a long part of this section to point out what they perceive as flaws of a single previous study covering mating signals used during swarming in the same mosquitoes. Instead, they should give more attention to describing their own work with better 
clarity in the last paragraph, as usually done when closing the introductory text. Please keep most of your perspectives on the methodological flaws of Mozuraitis and collabs. for the discussion section.

\section{Material and Methods}

According to the authors "Colonies originated from wild gravid females collected in inhabited human dwellings from Burkina Faso, in 2017 in Bama for An. coluzzii and in 2015 in Soumousso for An. gambiae." but they were "repeatedly refreshed with F1 from mosquito females caught in the wild".What does "repeatedly" mean? Once a year? It would be desirable to offer readers a clearer definition.

Line 370: please be specific and avoid ambiguous expressions such as "At "mosquito sunset time ${ }^{\prime \prime \prime \prime}$.

Line 371, the phrase "Males were checked for erected antennae, ensuring that change in light and dark rhythm, transport and electrophysiology laboratory conditions did not alter their physiological state for mating." needs edition. Firstly, instead of "light and dark rhythm" use the more adequate "light-dark cycles". Secondly, erected antennae should not be referred to as being equivalent to "physiological state for mating" as this is a mere proxy of what the authors look for that may indicate a motivation for mating, but would not grant unaltered mating physiology. For instance, you can state that this was used as a proxy for mating motivation without referring to whether the physiological state for mating was altered or not in mosquitoes.

\section{Results}

This section can be improved by making it more direct. There are many instances in which lack or presence of a compound in a particular sample are referred to, making the reader lose a clear path. As this reviewer understood, the results of the different experiments trying to sample male swarm-associated VOCs have shown that no significant chemical pattern could be established, suggesting that no VOCs are emitted by male swarms in these two species of Anopheles. Results should be better organized, so that the above conclusion could be stated at the beginning of the discussion, avoiding unnecessary details.

\section{Discussion}

Line 525: this phrase needs to be edited to make it straightforward: females were not attracted to air currents passed over male swarms.

Line 538: authors should tone this phrase down as negative EAD traces are by no means definitive proof of lack of olfactory sensory neurons (OSNs) in antennal sensilla. It should be noted that few OSNs may not be detectable in the complex signal recorded in EADs, but could very efficiently inform the brain of the presence of a certain ligand. As always in science, lack of response should not be strictly taken as a lack of signal, but suggested.

Line 574: I suggest toning down this phrase, as the authors themselves have said in the previous paragraph that the absence of presence does not prove a lack of male swarm pheromones.

\section{Reviewed by anonymous reviewer, 01 Jul 2021}

\section{Comments to the authors}

\section{Main comments}


I read the manuscript with great interest and I like the questions the authors addressed. I particularly like the olfactometer assay in which responses of female mosquitoes to swarms of males were tested. The authors investigated responses of two species of mosquitoes. I was impressed by the amount of effort placed in using different methodologies for volatile collection emitted by the male swarms. The overall goal of the study was to investigate if female mosquitoes respond to odours emitted by male swarms that would in this context be considered a sex pheromone(s).

Although I very much like the questions addressed and the olfactometer bioassay, I think some of the methodological details need to be clarified so we can better judge the quality of the research. Some of the conclusions are not expressed in the most accurate way in my view, and the manuscript needs to be largely improved prior to publication. The manuscript can benefit from a more streamlined introduction and the structure of M\&M section needs to be largely improved. The authors selectively analysed a few volatile compounds, and in this way the conclusions are limited. A more extensive analyses of the data I assume the authors have, and in combination with different statistical analyses could be of additional value for the manuscript, when details on the methods are clarified. I hope the authors will find my comments useful when reviewing their work.

Detailed comments

Introduction

Line 37-46: I would add all refs at the end of the sentence to facilitate the reading.

Line 55-57: What do the authors mean with visual markers, are these land markers in this context?

Line 60-71: this passage refers several times to markers, these markers, same markers. Can the authors please specify markers and the context? See earlier suggestion.

Line 72: Are there sex pheromones that are not volatile?

Lines 106-119: This paragraph could be significantly reduced and perhaps combined with the next one. The point here should be the connection of flight activity with the odour's cues, and nectar with odour cues - the text moves far away from the topic of the study.

Line 118-119: This is a confusing reference to 5 compounds that I assume are not the same 5 compounds the authors refer to in line 89.

Line 120: see previous, best to complement sentence by add such as these and these compounds

Line 130:" Bring to light the existence" should read "to investigate"

$M \& M$

This section could be largely improved by using more specific heading as well as headings and subheadings

Line 153: Please specify how often: yearly, $X$ times throughout the season...

Line 164-166: Not clear why this information is relevant for this paper 


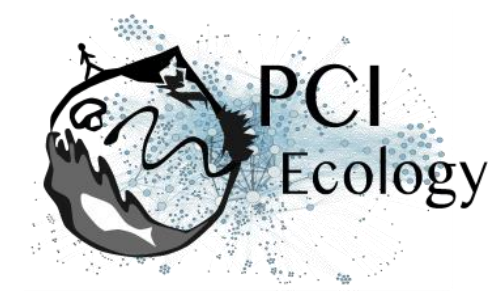

Lines 170-175: It would be great is dimensions could be added direct into the figure

Line 187: Bioassay is not sufficient. I suggest a general heading first referring to the objective of the experiment and then subheading on the set up, bioassay...

Line 188-191: How many replicates in total?

Lines 194-195. Glucose is not volatile thus there would be no odour bias. It is good that the authors add the glucose cup to the empty box as well so that humidity is similar in both boxes. I suggest to refers to these boxes as swarming boxes.

Line 197-201: This moving outside is not clear. Is that for natural light? So why there is need for additional artificial light?

Line 204: Previously to what?

Line 213: How many females were released at a time? Any evidences that females follow each other?

Line 223: 100 females per replicate? The replication info is a bit too spread through the text. Hard to follow.

Line 227: I guess the authors mean positional bias with "side effects".

Line 233: Not clear whether Burkina Faso is a lab, semi-field or field location/condition

Line 238-243: Not clear from where the air originated from. Did the authors used a cylinder with synthetic air, compressed air line or was air pumped air from the environment? It sounds like air was pumped from the environment, and this could explain why the authors do not see differences between treatments and controls. I don't think a charcoal filter is sufficient to eliminate compounds that are often present in the environment as are the compounds of interest here.

Table 1: From my experience with headspace collection the flow rate used is a too high. Did the authors do a breakthrough experiment, like placing a second tube just behind the tube used for collection to check if volatiles of interest a trapped in the second tube?

Line 253: It would be clearer for the general reader if the authors refer to the olfactometer swarming box

Line 254 vs. table 1: Please be consequent with unit format

Line 264: Carefully conditioned has no meaning for the general reader. Please, describe the procedure.

Line 268: The entire section is about VOC collection - from line 231 through line 319. I suggest making

subheadings more specific

Line 298-309: This passage could be reduced if the authors simply describe treatments and controls

Line 312: Why is this necessary? Is the cleaning procedure not efficient? 


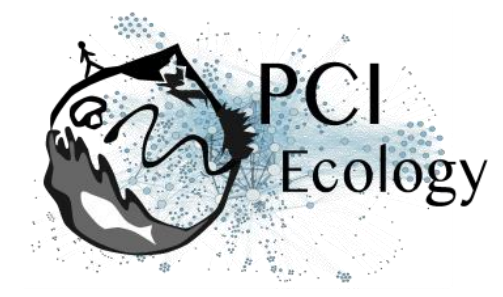

Lines 322-323: This should be the last sentence of the paragraph as it is not relevant for the general understanding of the procedure.

Lines 358-363 and Lines 407-425: Everything related to identification should be combined in one section.

Line 406: Would identification of volatile organic compounds be a better heading?

Line 428: last sentence of the paragraph?

Line 451: How many per replicates and per species? This was already not clear with M\&M section.

Line 452: two decimals is overdone.

Line 465-466: I think the authors mean that only quantitative differences were detected between controls and samples?

Line 483-496: Was the air pumped from the environment and perhaps charcoal filter not sufficient to get it clean and clear controls?

Results chemical analyses: It reads to me as if the authors selectively chose which compounds to quantify and identify. I think this approach limits the extent to which conclusions can be drawn. I expect the authors to have collected more VOCs in their blends. Even if not all compounds could be identified, I expect they could have been quantified. The authors also analysed VOCs individually. It would be interested to use some king of multivariate analyses too, though the largest issue I see here is the presence of the compounds of interest in the controls.

Line 517: it should read ...evidence is available

Line 531-533: Could it be that higher number of males was needed for females to detect the odour source in this set up?

\section{Discussion}

Line 521: I think the conclusions here need to be more accurately stated. The authors found no evidence that the volatile organic compounds quantified in this study could be exploited by females as a sex pheromone. I expect the authors to have collected more VOCs in their blends than they have chosen to quantify. Even if not all compounds could be identified, I expect they could have been quantified. Multivariate approaches would also be to address differences in the blends. 